\title{
Expression profiles of HER2, EGFR, MET and FGFR2 in a large cohort of patients with gastric adenocarcinoma
}

\author{
Akiko Kawano Nagatsuma $\cdot$ Masaki Aizawa • \\ Takeshi Kuwata $\cdot$ Toshihiko Doi $\cdot$ Atsushi Ohtsu \\ Hirofumi Fujii $\cdot$ Atsushi Ochiai
}

Received: 5 February 2014/Accepted: 8 February 2014/Published online: 14 March 2014

(c) The International Gastric Cancer Association and The Japanese Gastric Cancer Association 2014

\begin{abstract}
Background Some tyrosine kinase receptors (RTKs) play critical roles in gastric cancer progression. Not only trastuzumab, but also several other agents targeting RTKs are being investigated for gastric cancer therapy. However, the simultaneous expression of multiple RTKs, which may interfere with the effectiveness of therapeutic agents, has not been evaluated in a large cohort with gastric adenocarcinoma (GAC).

Methods We performed a tissue microarray analysis in 950 patients with GAC who underwent a gastrectomy without preoperative chemotherapy. The protein expressions of HER2, EGFR, MET and FGFR2 were evaluated using immunohistochemistry, and the gene amplifications of HER2, EGFR and MET were examined using dual-color in situ hybridization.
\end{abstract}

Electronic supplementary material The online version of this article (doi:10.1007/s10120-014-0360-4) contains supplementary material, which is available to authorized users.

A. K. Nagatsuma $\cdot$ T. Kuwata $\cdot$ A. Ochiai $(\bowtie)$

Division of Pathology, Research Center for Innovative

Oncology, National Cancer Center Hospital East,

6-5-1 Kashiwanoha, Kashiwa-city, Chiba 277-8577, Japan

e-mail: aochiai@east.ncc.go.jp

\section{A. K. Nagatsuma $\cdot$ H. Fujii}

Division of Medical Oncology, Jichi University School

of Medicine, 3311-1 Yakushiji, Shimotsuke-city,

Tochigi 329-0498, Japan

\section{Aizawa}

Division of Surgery, Niigata Cancer Center Hospital,

2-15-3 Kawagishicho, Niigata-city, Niigata 951-8566, Japan

T. Doi · A. Ohtsu

Division of Gastroenterology, National Cancer Center Hospital

East, 6-5-1 Kashiwanoha, Kashiwa-city, Chiba 277-8577, Japan
Results The frequency of overexpression was $11.8 \%$ for HER $2,23.5 \%$ for EGFR, $24.9 \%$ for MET and $31.1 \%$ for FGFR2. Whereas strong staining for each of the RTKs was heterogeneous, tumors with homogeneously strong staining areas often exhibited gene amplification. Strong EGFR expression was significantly associated with a poor outcome, but no prognostic correlations were observed in other RTKs. The overexpression of single and multiple RTKs was observed in 40.4 and $22.7 \%$ of the cases, respectively. HER2, EGFR, MET and FGFR2 predominance was observed in 10.1, 13.9, 16.1 and $22.9 \%$ of the GACs, respectively.

Conclusions Approximately two-thirds of patients with GAC exhibited the expression of at least one RTK and would be candidates for targeted therapies. Moreover, one-third of at least one RTK overexspressing cases showed multiple RTKs expression. Our results may be useful for selecting the most suitable patients for each targeted therapy.

Keywords Receptor protein-tyrosine kinases . Immunohistochemistry · Gene amplification · Prognosis . Molecular targeted therapy

\section{Introduction}

Gastric cancer is one of the most common malignant tumors worldwide. Although its incidence is decreasing, gastric cancer is still the second most frequent cause of cancer-related death in Japan [1,2]. The prognosis of patients with advanced gastric cancer remains poor, and the median survival time of patients with inoperable and/or gastric cancer is $<1$ year [3, 4].

Nowadays, the molecular mechanisms underlying tumorigenesis, proliferation, progression and drug resistance in 
gastric cancer are being elucidated. Several tyrosine kinase receptors (RTKs), such as the human epidermal growth factor receptor 2 (HER2), epidermal growth factor receptor 1 (EGFR), MET and fibroblast growth factor receptor 2 (FGFR2), are overexpressed in gastric cancer, and targeted therapies involving these molecules have been developed. The ToGA (Trastuzumab for Gastric Cancer) trial has shown that trastuzumab improves the survival of patients with HER2 protein-overexpressing and/or HER2 geneamplifying advanced gastric cancer [5]. Although the addition of cetuximab or panitumumab to chemotherapy in non-molecularly selected gastric cancer patients did not improve survival [6, 7], a phase 2 study has shown a possible improvement in the survival of the patient subset with high EGFR expression who were treated with nimotuzumab, a humanized monoclonal antibody to EGFR [8]. At present, a phase 3 nimotuzumab trial (ENRICH study) in patients with EGFR-overexpressing advanced gastric and gastroesophageal junction cancer is ongoing. Rilotumumab is a humanized monoclonal antibody to hepatocyte growth factor that inhibits signaling through the MET receptor. A phase 2 study of rilotumumab in advanced gastric cancer patients showed that treatment with rilotumumab in combination with chemotherapy improved the survival of the patient subset with high MET protein-expressing tumors [9]. A following phase 3 rilotumumab trial (RILOMET-1) in patients with advanced MET-positive gastric or gastroesophageal junction adenocarcinoma is ongoing [10]. Some new agents inhibiting FGFR2 signaling have been developed for solid tumors, including gastric cancer [11]. Several reports have examined the relation between clinical factors, pathological features, and prognosis and the protein overexpression or gene amplification of these RTKs. However, the expression and gene amplification profiles of these promising RTKs for gastric cancer treatment have not been simultaneously evaluated in a large cohort.

In the present study, we evaluated the expressions of HER2, EGFR, MET and FGFR2 proteins and the gene amplification of HER2, EGFR and MET in a large number of gastric cancers using tissue microarrays (TMAs), including expression ratios and distributions of all four RTKs with analyses in association with clinical outcomes were examined.

\section{Materials and methods}

Patients and data collection

There were 1,001 consecutive patients with gastric adenocarcinoma (GAC) who underwent surgery at the National Cancer Center Hospital East between January

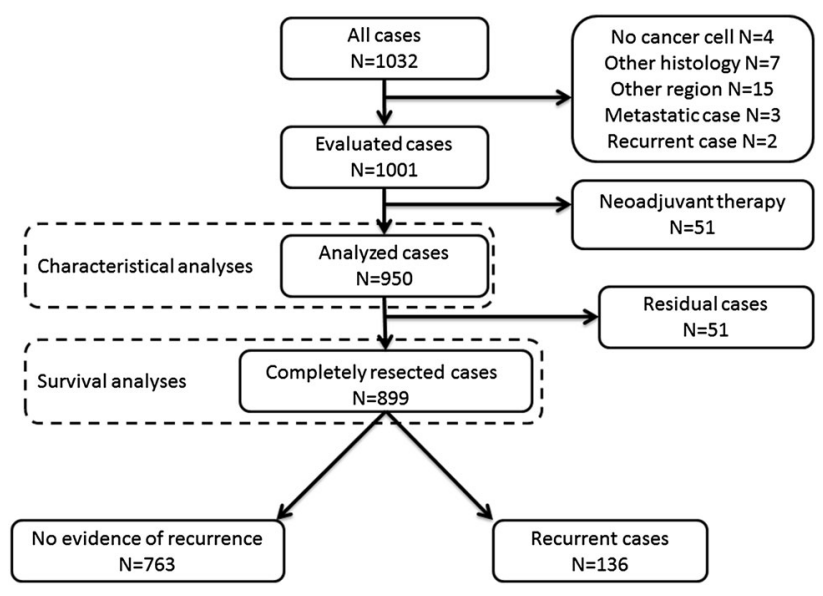

Fig. 1 Study schema

2003 and July 2007. A total of 950 patients with GAC with no systemic chemotherapy before surgery were enrolled in this study (Fig. 1). The medical records were reviewed to obtain the clinical parameters of the patients, including age, sex, stage, recurrence and survival outcomes. A pathological report and hematoxylin and eosin (H\&E)-stained slides were reviewed for the tumor parameters, including tumor localization, histopathology, depth of tumor invasion and capillary invasion status, such as lymphatic and venous invasion and lymph node metastasis. The staging was conducted according to the Japanese classification of gastric carcinoma, third English edition [12]. The study protocol was approved by the institutional review board at the National Cancer Center.

Tissue microarray construction

Representative tumor areas were selected and marked on $\mathrm{H} \& \mathrm{E}$-stained slides for the construction of the tissue microarrays. A couple of 2.0-mm-diameter tumor cores were obtained from the same tissue block in each case using a manual tissue arrayer (Azumaya Ika Kikai, Tokyo, Japan). These cores were assembled in a tissue microarray format. Each tissue array block contained 48 cores, including two positioning cores of liver tissue.

Immunohistochemical staining

The primary antibodies used for immunohistochemistry (IHC) were the Ventana PATHWAY anti-HER2/neu (4B5) rabbit monoclonal antibody (Ventana, Tucson, AZ), CONFIRM anti-EGFR (3C6) mouse monoclonal antibody (Ventana), CONFIRM anti-Total c-MET (SP44) rabbit monoclonal antibody (Ventana) and rabbit polyclonal antiFGFR2 antibody (C-17, SC-122, Santa Cruz Biotechnology, CA). 
Formalin-fixed, paraffin-embedded array blocks were cut into 4- $\mu \mathrm{m}$ serial sections. HER2, EGFR and MET IHC were stained using a fully automated Ventana Benchmark ULTRA automated slide processing system (Ventana) according to the manufacturer's instructions.

For FGFR2 staining, the sections were deparaffinized in xylene, dehydrated with graded ethanol and then immersed in methanol with $0.3 \%$ hydrogen peroxidase for $20 \mathrm{~min}$ to block endogenous peroxidase. After being washed in tap water, the sections were placed in a $10 \mathrm{mmol} / \mathrm{l}$ citrate buffer solution. For antigen retrieval, the sections were heated at $125{ }^{\circ} \mathrm{C}$ for $30 \mathrm{~s}$ followed by $90{ }^{\circ} \mathrm{C}$ for $10 \mathrm{~s}$ in a pressure cooker (Pascal Pressurized Heating Chamber) and then cooled for $30 \mathrm{~min}$ at room temperature. After being washed three times with PBS, the sections were immersed in $2 \%$ normal swine serum in PBS to block nonspecific binding for $30 \mathrm{~min}$ at room temperature. Individual slides were then incubated overnight at $4{ }^{\circ} \mathrm{C}$ with anti-FGFR2 antibody. After being washed at least five times with PBS, the slides were incubated with a peroxidase-labeled polymer conjugated to goat anti-rabbit immunoglobulin (Dako EnVision+ System-HRP labelled polymer anti-rabbit, code K4002; Dako) for $30 \mathrm{~min}$ at room temperature. After extensive washing with $\mathrm{PBS}$, the color reaction was developed in $2 \% 3,3^{\prime}$-diaminobenzidine in $50 \mathrm{mmol} / \mathrm{l}$ Tris-buffer ( $\mathrm{pH} 7.6)$ containing $0.3 \%$ hydrogen peroxide for $3 \mathrm{~min}$. The sections were then counterstained with Meyer's hematoxylin. All the stained sections were dehydrated and mounted.

\section{Dual-color in situ hybridization}

The gene amplification of HER2, EGFR and MET was detected using the double-color ISH (DISH) technique using the Ventana Benchmark ULTRA automated slide processing system according to the manufacturer's instructions. HER2 DISH was performed using a fully automated INFORM HER2 Dual ISH DNA Probe Cocktail assay (Ventana). EGFR DNA, MET DNA and chromosome 7 were detected using the INFORM EGFR DNA Probe (Ventana), INFORM MET DNA Probe (Ventana) and INFORM CHROMOSOME 7 Probe (Ventana), respectively. To detect the HER2, EGFR and MET DNA probes, the ultraView SISH DNP kit (Ventana) was used. After the SISH signals had been developed, the RED ultraView ISH DIG detection kit (Ventana) was used to detect the chromosome 17 or 7 probes.

\section{Evaluation}

The staining intensity was graded on a scale of $0-3(0$, no staining; 1, faint staining; 2, weak or moderate staining; 3 , strong staining). Evaluation and scoring of the HER2, EGFR and MET IHC expressions were performed according to Hofmann's criteria [13]. This scoring system has been validated for use in gastric cancer: $0=$ staining or membrane reactivity in $<10 \%$ of cancer cells; $1+=$ membrane reactivity with an intensity score of 1 in $>10 \%$ of cancer cells or cancer cells with reactivity only in a part of their cell membrane; $2+=$ complete or basolateral membrane with an intensity score of 2 in $>10 \%$ of cancer cells; $3+=$ complete or basolateral membrane with an intensity score of 3 in $>10 \%$ of cancer cells. For FGFR2, we examined the nuclear and cytoplasmic staining patterns. We defined the scores for FGFR2 as follows: $0=$ staining reactivity in $<50 \%$ of cancer cells; $1+=$ cytoplasm and/or nuclear reactivity with an intensity score of 1 in more than $50 \%$ of the cancer cells; $2+=$ cytoplasm and/or nuclear reactivity with an intensity score of 2 in more than $50 \%$ of the cancer cells; $3+=$ cytoplasm and/or nuclear reactivity with an intensity score of 3 in more than $50 \%$ of the cancer cells. Representative images of corresponding IHC staining scores for each RTK are shown in the supplementary Fig. 1.

We evaluated the gene amplifications of HER2, EGFR and MET by counting at least 20 tumor nuclei within a hot spot using IHC staining. We defined HER2 gene amplification if the HER2/Chr17 ratio was $\geq 2.0$ or if the HER2 signals formed a cluster. The evaluation of EGFR and MET gene amplification was performed according to the University of Colorado Cancer Center criteria [14]. We identified gene amplification according to the presence of tight EGFR/MET gene clusters and a ratio of EGFR gene to chromosome of $\geq 2$ or $\geq 15$ copies of EGFR/MET per cell in $\geq 10 \%$ of analyzed cells.

An IHC score of $3+$ or a score of $2+$ plus HER2 gene amplification was defined as HER2 positive. In EGFR, MET and FGFR2, we defined an IHC score of $2+$ and $3+$ as positive. In multiple RTK-expressing cases, we classified the "predominant" RTK according to the following rules: the RTK with the strongest intensity of IHC staining, or the broadest positive area if multiple RTK expressing intensities were equal. We also classified overlapping situations into concurrently co-existing (overexpression of multiple RTKs in the same area) and exclusively existing (overexpression of multiple RTKs in mutually exclusive areas) patterns.

Statistical analysis

The statistical analysis was performed using SPSS 19.0 statistical software (SPSS Inc., Chicago, IL). Comparisons of categorical variables were tested using the chi-square test or Fisher's exact test. Overall survival (OS) was calculated from the date of surgery until death from any cause that was scored as an event. Patients who were alive were 
Table 1 Intratumoral heterogeneity of IHC strong staining

\begin{tabular}{llrrr}
\hline \multicolumn{1}{c}{ HER2 } & \multicolumn{1}{l}{ EGFR } & \multicolumn{1}{l}{ MET } & \multicolumn{1}{c}{ FGFR2 } \\
\hline IHC strong staining area & & & \\
$<10 \%$ & $11(10.6)$ & $26(27.4)$ & $93(60.0)$ & \\
$\geq 10 \%,<30 \%$ & $28(26.9)$ & $35(36.8)$ & $42(27.1)$ & $120^{\mathrm{a}}(81.1)$ \\
$\geq 30 \%,<80 \%$ & $29(27.9)$ & $28(29.5)$ & $15(9.7)$ & $26(17.6)$ \\
$\geq 80 \%$ & $36(34.6)$ & $6(6.3)$ & $5(3.2)$ & $2(1.4)$ \\
\hline
\end{tabular}

IHC immunohistochemistry

${ }^{\text {a }}$ Under $30 \%$ of FGFR2 IHC staining area

censored at the last follow-up date. The survival curves were derived from Kaplan-Meier estimates, and the curves were compared using log-rank tests and the Cox proportional hazard regression model. All the tests were twosided, and $P$ values of $<0.05$ were considered significant.

\section{Results}

Overexpression and gene amplification of four RTKs

The HER2 IHC score was 0 in 483 cases, $1+$ in 297 cases, $2+$ in 77 cases and $3+$ in 93 cases. The EGFR IHC score was 0 in 350 cases, $1+$ in 377 cases, $2+$ in 154 cases and $3+$ in 69 cases. The MET IHC score was 0 in 291 cases, $1+$ in 422 cases, $2+$ in 176 cases and $3+$ in 61 cases. The FGFR2 IHC score was 0 in 84 cases, $1+$ in 571 cases, $2+$ in 268 cases and $3+$ in 27 cases. Among the 77 cases with a HER2 IHC intensity score of $2+, 19$ cases had gene amplification when examined using DISH. Only 23 (2.4\%) and $12(1.3 \%)$ patients exhibited EGFR and MET gene amplification, respectively.

Table 1 shows the intratumoral expression ratio of each RTK in cases with an intensity score 3 staining area. Onethird of the samples with strong staining for HER2 were homogeneously stained; however, the samples with strong staining for EGFR, MET and FGFR2 exhibited highly heterogeneous staining. Table 2 shows the correlation between gene amplification and the intratumoral heterogeneity of areas with strong IHC staining. Patients with gene amplification tended to have strongly staining tumors with a homogeneous staining pattern. In patients with tumors not amplified EGFR or MET gene, the gene status of strongly staining areas with a homogeneous pattern was polysomic.

Correlation between the positivity of each RTK and clinicopathological factors

Table 3 shows the clinicopathological data for each RTKpositive case. The patients with HER2-positive tumors
Table 2 Correlation with gene amplification and intratumoral heterogeneity of IHC strong staining

\begin{tabular}{lccl}
\hline & $\begin{array}{l}\text { HER2 gene } \\
\text { amplification }\end{array}$ & $\begin{array}{l}\text { EGFR gene } \\
\text { amplification }\end{array}$ & $\begin{array}{l}\text { MET gene } \\
\text { amplification }\end{array}$ \\
\hline $\begin{array}{l}\text { Number of } \\
\text { patients }(\%)\end{array}$ & 90 & 23 & 12 \\
IHC strong staining area & & \\
$<10 \%$ & $6(54.5)$ & $3(11.5)$ & $5(5.4)$ \\
$\geq 10 \%,<30 \%$ & $19(67.9)$ & $5(14.3)$ & $1(2.4)$ \\
$\geq 30 \%,<80 \%$ & $29(100)$ & $10(35.7)$ & $4(26.7)$ \\
$\geq 80 \%$ & $36(100)$ & $5(83.3)$ & $2(40.0)$ \\
\hline
\end{tabular}

The number in parentheses is the percentage of gene amplifying cases in each IHC strong staining area category (Table 1)

IHC immunohistochemistry

were elderly and predominantly male. The HER2-positive GACs were a more differentiated tumor type (papillary and tubular adenocarcinoma) and were more frequently associated with venous invasion and regional lymph node metastasis than the HER2-negative ones. The EGFR-positive GACs were of more poorly differentiated adenocarcinoma solid-type (por1) histopathology and an advanced tumor stage, and were more frequently associated with vascular invasion than the EGFR-negative ones. METpositive GACs were a more differentiated tumor type (tubular adenocarcinoma) and had an earlier tumor stage than the MET-negative ones. The FGFR2-positive GACs were more frequently associated with vascular invasion and had a more advanced tumor stage than the FGFR2negative ones. Table 4 shows the sites of recurrence and metastasis in patients for each RTK-positive case. HER2positive tumors were significantly associated with the recurrence of liver and intrathoracic organ metastases (lung, mediastinal lymph node, pleura and lymphangiosis). Patients with FGFR2-positive tumors had a greater chance of peritoneal recurrence and a smaller chance of abdominal lymph node metastasis.

\section{Clinical outcomes according to each RTK}

We performed survival analyses in the 899 patients who had undergone a complete resection. The median follow-up time was 5.01 years (range, 3 days to 10.5 years), and 169 deaths were observed. The Kaplan-Meier curves for OS are shown in Fig. 2. Patients with EGFR- and FGFR2-overexpressing tumors had shorter survival times than those with non-overexpressing tumors (log rank $P<0.001$ and 0.019 , respectively). Conversely, patients with METoverexpressing tumors had longer survival times than those with non-overexpressing tumors ( $\log \operatorname{rank} P=0.006$ ). For HER2, no significant differences in survival between overexpressing and non-overexpressing cases were seen. 


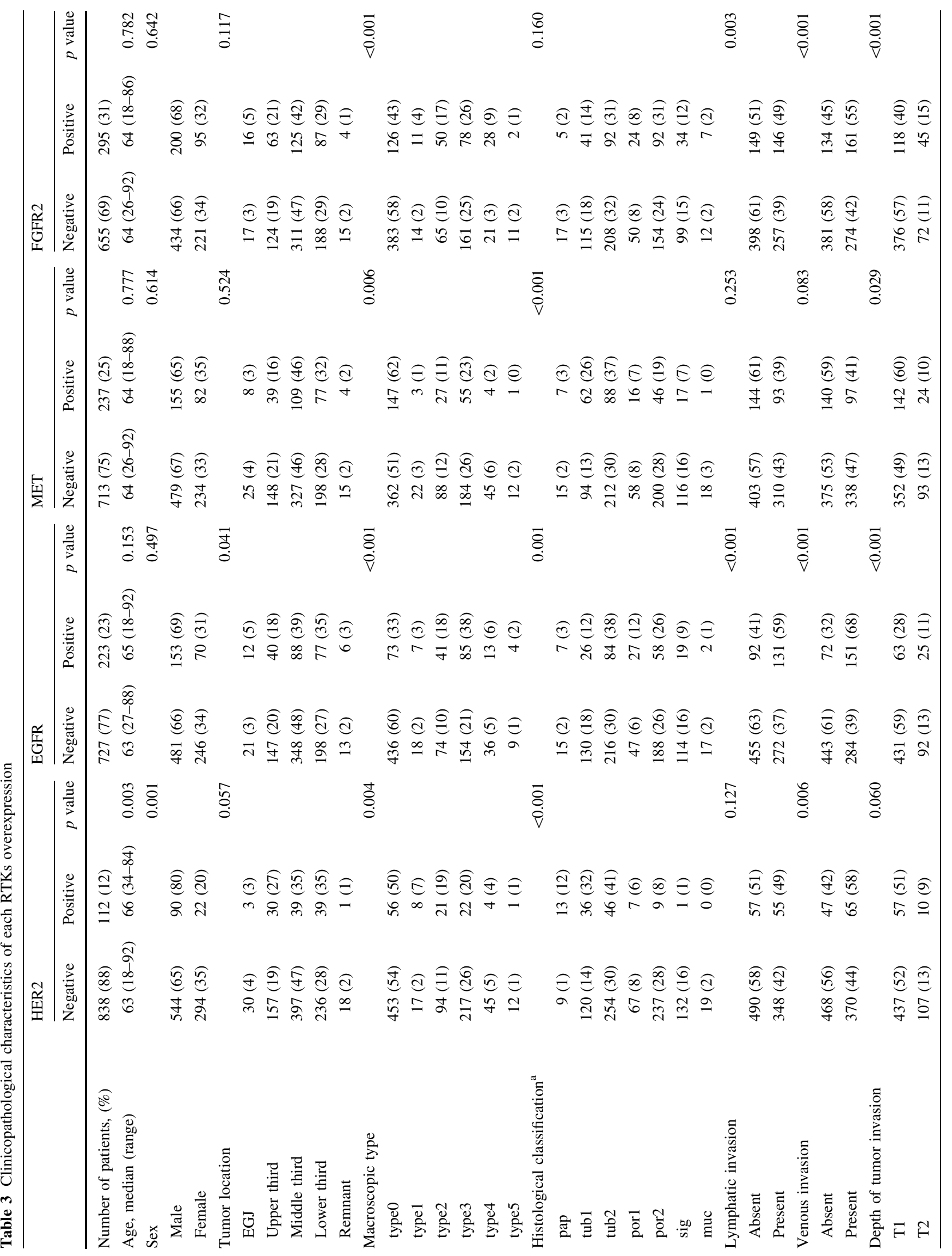




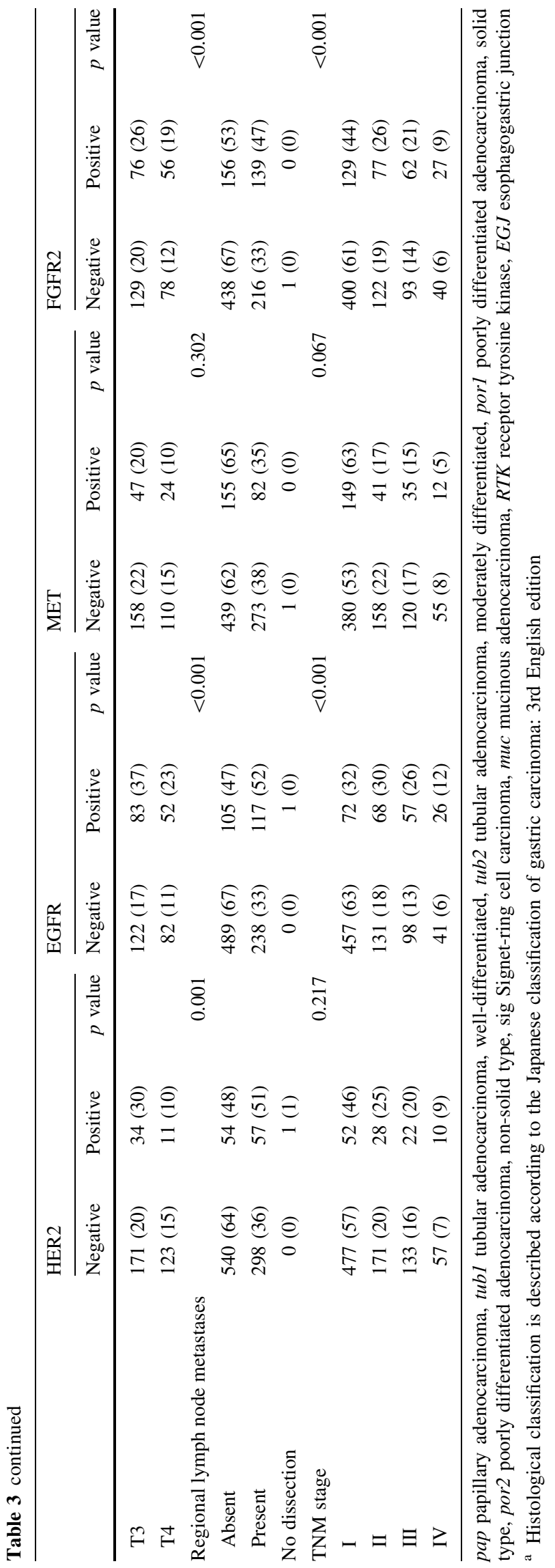

Only the patients with EGFR score 3+ tumors had significantly poorer outcomes than those with EGFR score 0 tumors (hazard ratio, 1.762; $95 \%$ confidence interval, 1.076 to $2.887 ; P=0.024$ ) when examined using a multivariate proportional regression analysis that included sex, age, vascular invasion status, histopathology, TNM stage and adjuvant chemotherapy. The positivity of each RTK except EGFR or multiple RTKs was not significantly correlated with patient outcome in a multivariate Cox regression model (data not shown).

\section{Distributions of four RTKs}

In 950 analyzed cases, no positivity for any of the four RTKs was seen in 351 (36.9\%) samples. Three hundred eighty-four (40.4\%) samples were positive for one RTK: 33 (3.5\%) samples overexpressed HER2 only, 84 (8.8\%) overexpressed EGFR only, $102(10.7 \%)$ overexpressed MET only and $165(17.4 \%)$ overexpressed FGFR2 only. Furthermore, $164(17.3 \%)$ samples were positive for 2 RTKs, $50(5.3 \%)$ were positive for 3 RTKs, and 1 (0.1\%) was positive for all 4 RTKs (Fig. 3a). The images of tumors with all four RTKs are shown in supplementary Fig. 2.

Of the 215 tumors with positive expressions for two or more RTKs, $100(46.5 \%)$ exhibited the strong (3+) expression of one RTK and the moderate $(2+)$ expression(s) of the other RTK(s) (strongest intensive pattern). Based on the predominant expression, 43 cases were classified as predominantly HER2-overexpressing, 29 cases were classified as predominantly EGFR-overexpressing, 21 were classified as predominantly METoverexpressing, and 7 were classified as predominantly FGFR2-overexpressing. Among the 115 (53.5\%) tumors with expression patterns of equal intensity, 2 simultaneously exhibited the expressions of three RTKs with a strong intensity (HER2/EGFR/FGFR2 and EGFR/MET/ FGFR2), 33 simultaneously exhibited the expressions of two RTKs with a strong intensity (6 with HER2/EGFR, 19 with HER2/MET, 5 with EGFR/MET, and 1 each with HER2/FGFR2, EGFR/FGFR2, and MET/FGFR2), and 80 simultaneously exhibited the expressions of all the RTKs with a moderate intensity. Based on the predominant expression in the broadest positive area, 20 cases were categorized as predominantly HER2, 19 were categorized as predominantly EGFR, 30 were categorized as predominantly MET, and 46 were categorized as predominantly FGFR2. The distribution of the overexpressions of multiple RTKs with equal intensity patterns was identified as a concurrently co-existing pattern in $76(66.1 \%)$ cases and an exclusively existing pattern in $39(33.9 \%)$ cases (Fig. 4a-d). Taken together, the positive cases were categorized as HER2-predominant tumors in 96 cases $(10.1 \%)$, EGFR-predominant tumors in 132 (13.9\%) 
Table 4 Sites of recurrence or metastasis in patients

\begin{tabular}{|c|c|c|c|c|c|c|c|c|c|c|c|c|}
\hline & \multicolumn{3}{|l|}{ HER2 } & \multicolumn{3}{|l|}{ EGFR } & \multicolumn{3}{|l|}{ MET } & \multicolumn{3}{|l|}{ FGFR2 } \\
\hline & Negative & Positive & $p$ value & Negative & Positive & $p$ value & Negative & Positive & $p$ value & Negative & Positive & $p$ value \\
\hline $\begin{array}{l}\text { Recurrence } \\
\text { cases, N (\%) }\end{array}$ & $114(84)$ & $22(16)$ & & $81(60)$ & $55(40)$ & & $113(83)$ & $23(17)$ & & $81(60)$ & $55(40)$ & \\
\hline Liver & & & 0.043 & & & 0.819 & & & 0.067 & & & 0.203 \\
\hline Absent & $82(72)$ & $11(50)$ & & $56(69)$ & 37 (67) & & $81(72)$ & $12(52)$ & & $52(64)$ & $41(75)$ & \\
\hline Present & $32(28)$ & $11(50)$ & & $25(31)$ & $18(33)$ & & $32(28)$ & $11(48)$ & & $29(36)$ & $14(25)$ & \\
\hline Peritoniae & & & 0.136 & & & 0.936 & & & 0.101 & & & 0.014 \\
\hline Absent & $69(61)$ & 17 (77) & & $51(63)$ & $35(64)$ & & $68(60)$ & $18(78)$ & & $58(72)$ & $28(51)$ & \\
\hline Present & 45 (39) & $5(23)$ & & $30(37)$ & $20(36)$ & & $45(40)$ & $5(22)$ & & $23(28)$ & 27 (49) & \\
\hline $\begin{array}{l}\text { Abdominal } \\
\text { lymph node }\end{array}$ & & & 0.327 & & & 0.819 & & & 0.894 & & & $<0.001$ \\
\hline Absent & 76 (67) & $17(77)$ & & $56(69)$ & 37 (67) & & 77 (68) & $16(70)$ & & $46(57)$ & $47(85)$ & \\
\hline Present & 38 (33) & $5(23)$ & & $25(31)$ & $18(33)$ & & $36(32)$ & 7 (30) & & $35(43)$ & $8(15)$ & \\
\hline $\begin{array}{l}\text { Intrathoracic } \\
\text { organ }^{\mathrm{a}}\end{array}$ & & & 0.006 & & & 0.531 & & & 0.470 & & & 0.270 \\
\hline Absent & $110(96)$ & 17 (77) & & 76 (94) & $51(93)$ & & $106(94)$ & $21(91)$ & & 77 (95) & $50(91)$ & \\
\hline Present & $4(4)$ & $5(23)$ & & $5(6)$ & $4(7)$ & & $7(6)$ & $2(9)$ & & $4(5)$ & $5(9)$ & \\
\hline $\begin{array}{l}\text { Bone, bone } \\
\text { marrow }\end{array}$ & & & 0.234 & & & 0.300 & & & 0.217 & & & 0.585 \\
\hline Absent & $106(93)$ & $22(100)$ & & 75 (93) & $53(96)$ & & $105(93)$ & $23(100)$ & & $76(94)$ & $52(95)$ & \\
\hline Present & $8(7)$ & $0(0)$ & & $6(7)$ & $2(4)$ & & $8(7)$ & $0(0)$ & & $5(6)$ & $3(5)$ & \\
\hline Local & & & 0.496 & & & 0.626 & & & 0.146 & & & 0.374 \\
\hline Absent & $105(92)$ & $21(95)$ & & 75 (93) & $51(93)$ & & $103(91)$ & $23(100)$ & & 76 (94) & $50(91)$ & \\
\hline Present & $9(8)$ & $1(5)$ & & $6(7)$ & $4(7)$ & & $10(9)$ & $0(0)$ & & $5(6)$ & $5(9)$ & \\
\hline $\begin{array}{l}\text { Stage } 4 \text { cases, } \\
N(\%)\end{array}$ & $57(85)$ & $10(15)$ & & $41(61)$ & 26 (39) & & $55(82)$ & 12 (18) & & $40(60)$ & 27 (40) & \\
\hline Liver & & & 0.537 & & & 0.598 & & & 0.574 & & & 0.362 \\
\hline Absent & $48(84)$ & $9(90)$ & & $35(85)$ & $22(85)$ & & $47(85)$ & $10(83)$ & & $33(83)$ & $24(89)$ & \\
\hline Present & $9(16)$ & $1(10)$ & & $6(15)$ & $4(15)$ & & $8(7)$ & $2(9)$ & & 7 (18) & $3(11)$ & \\
\hline Peritoniae & & & 0.327 & & & 0.640 & & & 0.534 & & & 0.205 \\
\hline Absent & $21(37)$ & $5(50)$ & & $15(37)$ & $11(42)$ & & 21(19) & $5(22)$ & & $18(45)$ & $8(30)$ & \\
\hline Present & $36(63)$ & $5(50)$ & & $26(63)$ & $15(58)$ & & $34(30)$ & $7(30)$ & & $22(55)$ & $19(70)$ & \\
\hline $\begin{array}{l}\text { Abominal } \\
\text { lymph node }\end{array}$ & & & 0.540 & & & 0.382 & & & 0.408 & & & 0.481 \\
\hline Absent & $42(74)$ & 7 (70) & & $31(76)$ & $18(69)$ & & $41(36)$ & $8(35)$ & & $28(70)$ & $21(78)$ & \\
\hline Present & $15(26)$ & $3(30)$ & & $10(24)$ & $8(31)$ & & $14(12)$ & $4(17)$ & & $12(30)$ & $6(22)$ & \\
\hline
\end{tabular}

${ }^{a}$ Intrathoracic organs include lung, mediastinal lymph node, pleura and lymphangiosis

$N$ number of patients

cases, MET-predominant tumors in $153(16.1 \%)$ cases, and FGFR2-predominant tumors in 218 (22.9\%) cases (Fig. 3b).

On the other hand, five patients had multiple RTKs amplified in their GAC cells (Fig. 4e-h). In two cases, both the HER2 and EGFR genes were amplified in the same cancer cells (concurrently co-existing pattern). In another three cases, the HER2/EGFR or EGFR/MET genes were amplified in discrete cancer cells obtained from the same core (mutually exclusive pattern).

\section{Discussion}

Here, we reported that $63.1 \%$ of resected GACs expressed at least one of four RTKs that are regarded as being potentially druggable targets for the molecular targeting agents in advanced GAC patients. Moreover, $22.7 \%$ of the cases expressed multiple RTKs simultaneously in the same sample. Drescher et al. [15] reported the expression patterns of six RTKs, including EGFR, using IHC in GAC, and only $2 \%$ of the samples in their series did not exhibit RTK expression. 

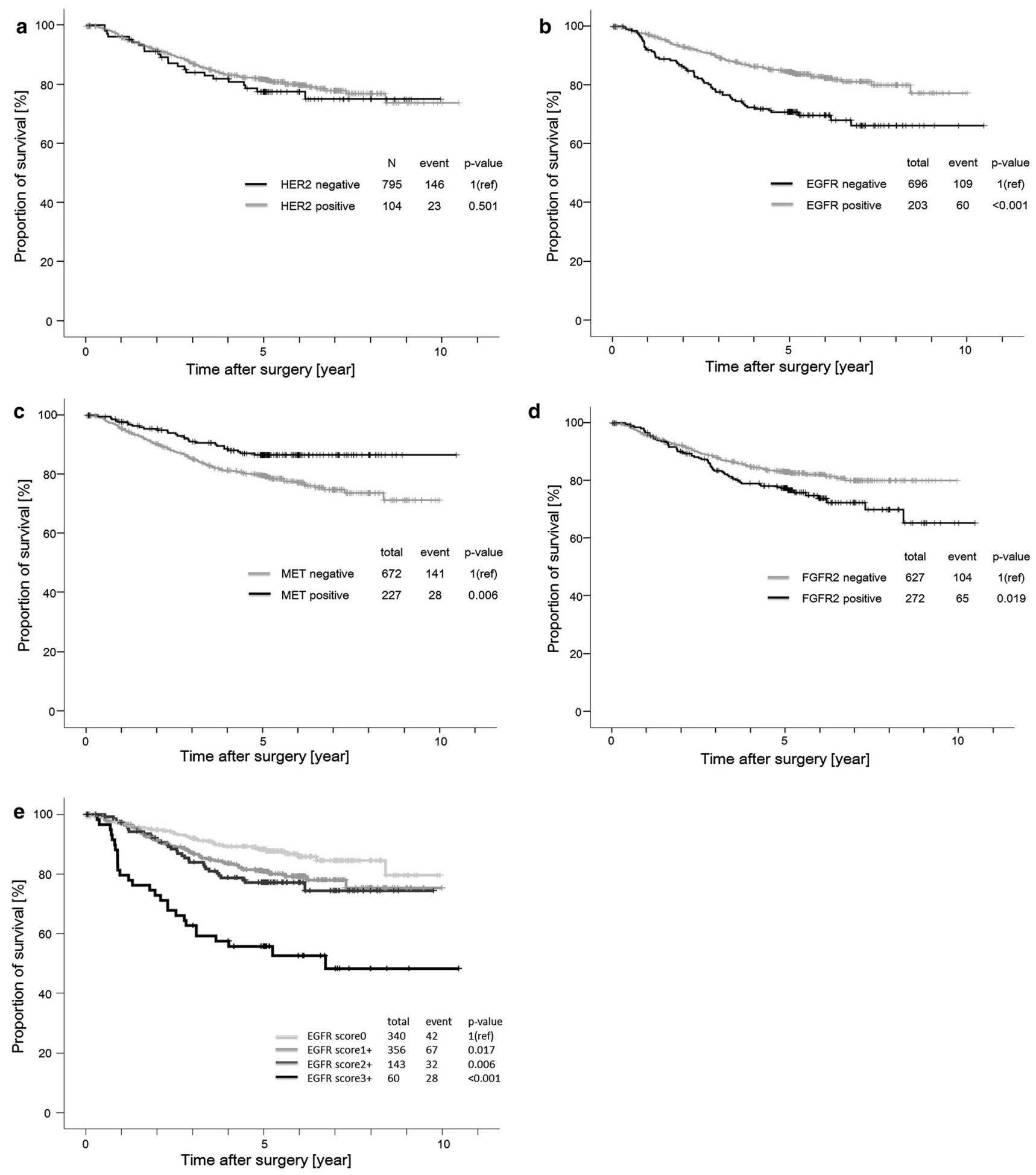

Fig. 2 Kaplan-Meier survival analyses of overall survival in HER2overexpressed tumors (a), EGFR-overexpressed tumors (b), METoverexpressed tumors (c), and FGFR2-overexpressed tumors (d).

e shows survival curves according to EGFR immunohistochemical score using Kaplan-Meier survival analysis

Guo et al. [16] reported that $88 \%$ of gastric cancer tissues showed the upregulation of at least one of four proteins, including MET and FGFR2, while $20 \%$ of them

overexpressed all four proteins. Other reports using IHC have shown the coexpression of MET/HER2 in $7.8 \%$ and the coexpression of EGFR/HER2 in $15 \%$ and $7 \%$ of gastric 

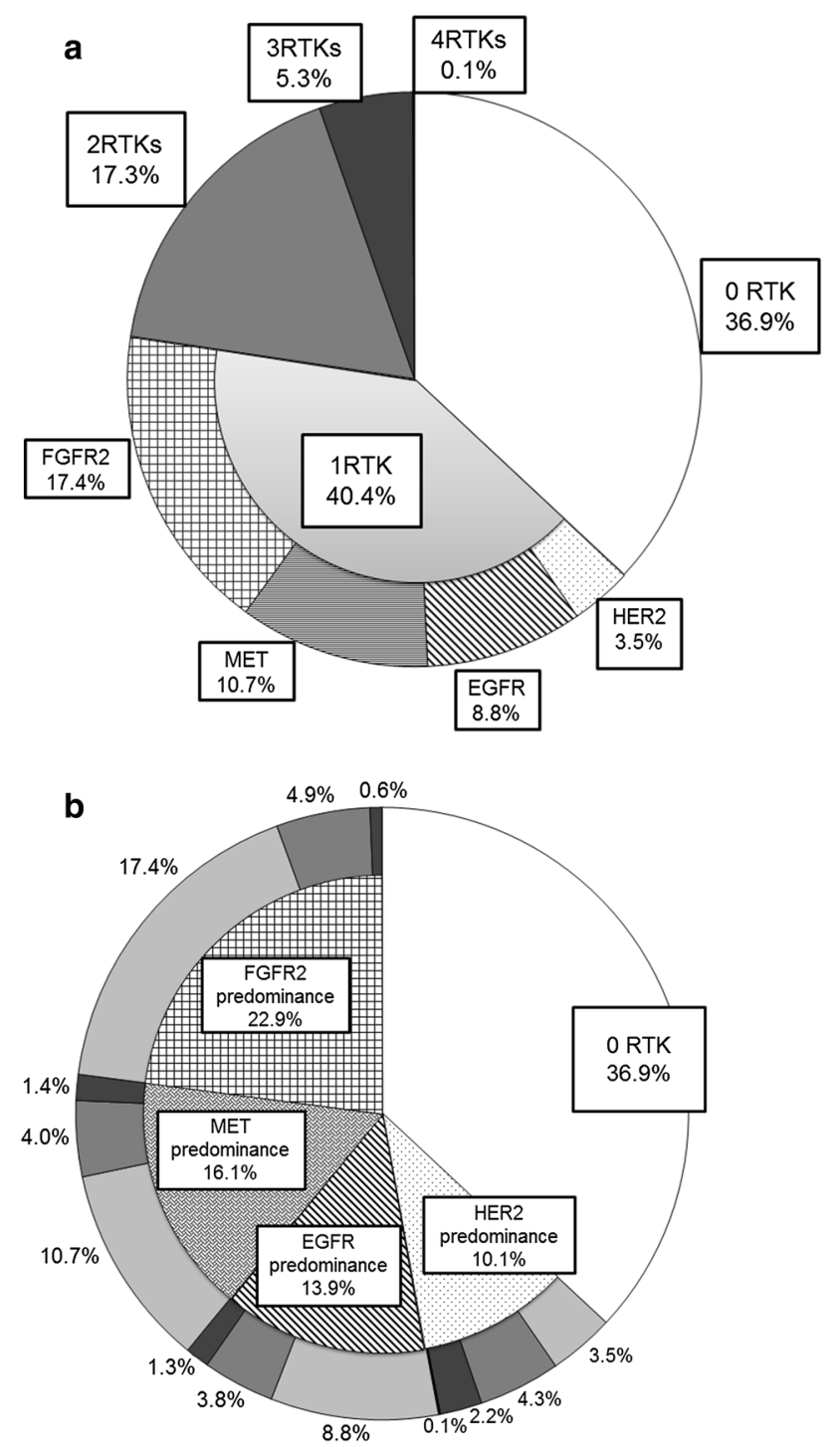

Fig. 3 a shows the profile of the number of positive RTK in gastric adenocarcinoma. $\mathbf{b}$ shows the profile of predominant RTK in gastric adenocarcinoma. The segment in bright gray, gray, bright gray and black denotes the sample with positivity of one RTK, two RTKs, three RTKs and all four RTKs, respectively. The HER2-predominant tumor is associated with a high frequency of overexpression of multiple RTKs, whereas the other RTK (EGFR, MET and FGFR2)predominant tumors are associated with a high frequency of overexpression of single RTK

cancers [17-19]. On the other hand, Lennerz et al. reported that HER2, MET and EGFR amplifications assessed using fluorescence in situ hybridization (FISH) were almost mutually exclusive events in gastroesophageal cancer [20]. Deng et al. [21] also described that FGFR2, HER2, EGFR and MET amplification as assessed using an SNP array were mutually exclusive events. Other reports have shown that FGFR2 amplification was found to be mutually exclusive with HER2 or HER2/MET amplifications [22, 23]. In summary, although multiple proteins of RTKs were overexpressed simultaneously, gene amplification or a gain in the gene copy number of an RTK was an exclusive event. Our high-volume study showed the parallel occurrence of these phenomena. Previous reports also have shown that only a portion of protein expression cases exhibit gene amplification [24-26], so gastric cancers with RTK protein overexpression might be derived not only from RTK gene amplification, but also via other mechanisms.

Regarding treatment, since gastric cancers with strong or moderate overexpression of HER2 protein plus gene amplification are associated with the efficacy of trastuzumab, gene amplification does not necessarily surpass protein expression with regard to predicting the effect of targeted therapy with antibodies [5]. On the other hand, lapatinib [a dual anti-EGFR and anti-HER2 tyrosine kinase inhibitor (TKI)] did not improve the survival of patients with HER2-positive gastric cancer [27, 28]. For optimal targeted therapy, we might need to detect each optimal target to each drug using simple techniques whenever possible. Our results may help to identify candidates whose outcome could be improved by treatment with apposite antibody therapy.

Drug resistance in molecular targeted therapy has been identified as playing a role in the alternative activations of other RTKs. In MET-addicted gastric cancer, activation of HER family members was poorly responsive to MET inhibitor [29]. In HER2-overexpressing breast cancer, the insulin-like growth factor receptor, other human epidermal growth factor receptor family members (EGFR, HER3) and MET overexpression might be critical for treatmentacquired resistance to trastuzumab [30-32]. In lung cancer, drug resistance to EGFR-TKI was associated with MET amplification [33, 34]. Moreover, MET activation was shown to lead to resistance to lapatinib in HER2-amplified gastric cancer [35]. Tumors develop more aggressively resulting in additional proliferation, invasion, metastasis and drug resistance, and the activation of multiple RTK pathways might be an effective means of promoting the survival of cancer cells. Thus, multiple RTK-expressing tumors may give us some suggestions for future strategies involving targeted therapy for gastric cancer.

In this study, we demonstrated the clinicopathological features and prognostic values associated with the overexpression of four RTKs evaluated by experts in GAC. Regarding HER2 and EGFR, our results were similar to those of previous reports with regard to the clinicopathological features and prognosis of each RTK-overexpressing tumor [26, 36-41]. However, contrary to previous reports, MET-overexpressing GACs tended to be associated with a rather favorable outcome in our study [25, 42, 43]. In addition, while we did not detect a correlation between FGFR2-overexpressing tumors and a specific histology, as previously reported, our FGFR2 IHC assay was able to 

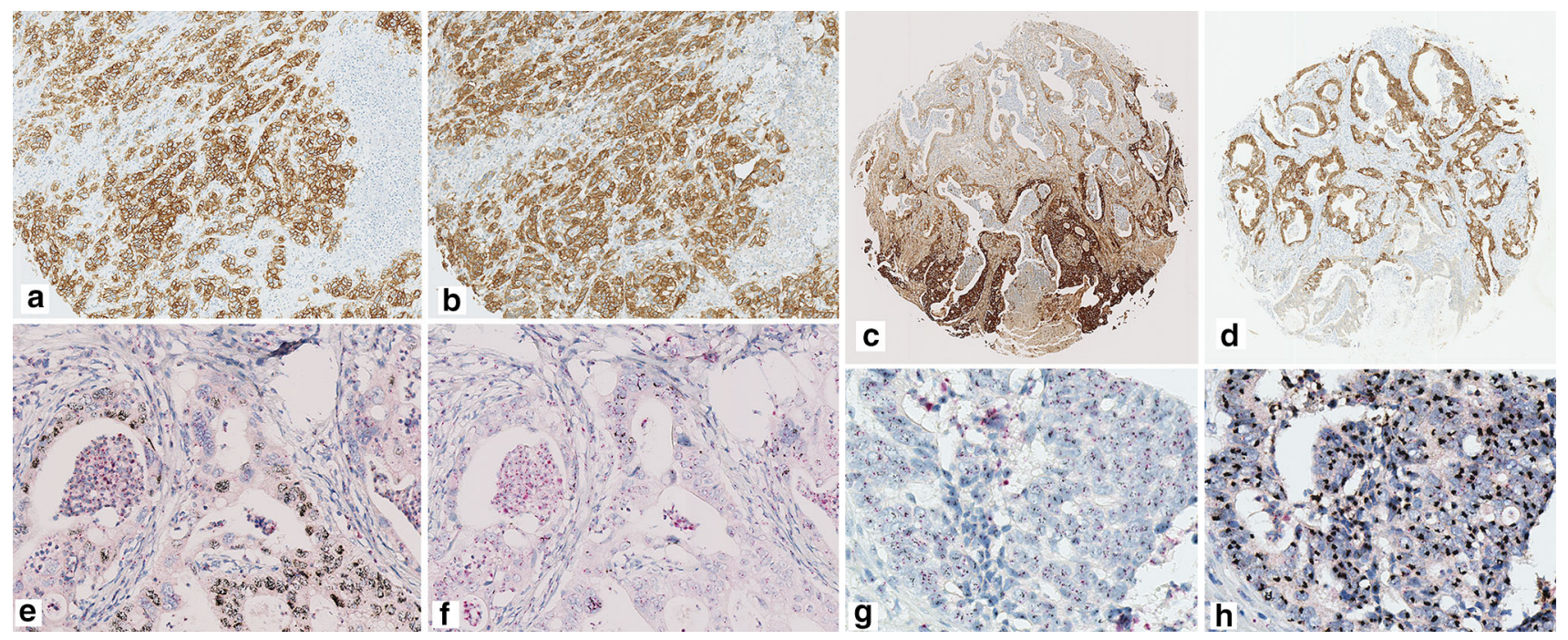

Fig. 4 Representative images of overexpressing or amplifying pattern of multiple RTKs. A concurrently co-existing pattern using immunohistochemical (IHC) assay of HER2 (a) and MET (b) is shown $(\times 100)$. An exclusively existing pattern using IHC assay of EGFR (c) and MET (d) is shown $(\times 25)$. On lower half of area, this tumor exhibits the strong expression of EGFR, but the weak expression of MET. Conversely, this tumor exhibits the strong

identify aggressive tumors [44, 45]. Except for HER2, some of the differences between previous reports and our results were attributed to the lack of standardized IHC protocols (antibody and staining protocol) and evaluations. Differences in the sample size and candidates themselves were also major reasons. Regarding MET, both the membranous and cytoplasmic expressions of MET, and not the membranous expression only, have been suggested to be suitable predictors of outcome [43]. Not only cytoplasmic expression, but also the nuclear expression of FGFR2 has been previously investigated in breast cancer and lung cancer [46, 47]. In breast cancer, Martin et al. suggested that the nuclear localization of FGFR2 might have an effect on the prevention of malignant changes. In lung squamous cell carcinoma, whereas the cytoplasmic overexpression of FGFR2 was correlated with a better outcome, the nuclear overexpression was correlated with a poorer outcome. Behrens et al. suggested that the nuclear localization of FGFR2 from the cytoplasm might lead to aggressive cancer changes. We hypothesized that not only the cytoplasmic expression, but also the nuclear expression of FGFR2 in gastric cancer may have an important role in cancer malignancy. On the other hand, both the nuclear and cytoplasmic overexpression of FGFR2 can be described as features of "FGFR2-enriched gastric cancer," but antibodies targeting FGFR2 do not seem to attack nuclear FGFR2. These findings suggest that tools for selecting candidates for antibody therapy may differ from those used to select specific tumors dominated by a specific oncogene. expression of MET but the moderate expression of EGFR on upper half of area. The EGFR (e) or MET (f) gene amplification is coincident with the area which EGFR or MET protein has strongly overexpressed $(\times 200)$. A concurrently co-existing pattern using dualcolor in situ hybridization (DISH) assay of HER2 (g) and EGFR (h) is shown. Both HER2 and EGFR are co-amplified on upper half of area $(\times 400)$.

Although we used TMAs to investigate RTK expression in this study, the percentage of IHC staining and the heterogeneity of each RTK expression might not have been evaluated accurately. TMA is a very useful tool for analyzing a large number of cases, but the results cannot escape the problems of sampling biases. One of the reasons for the discrepancy between TMA and whole tissue sections is thought to be the heterogeneity of IHC overexpression, and investigators have been recommended to obtain more than one core in each case [37]. We obtained two cores from different areas to evaluate the tissue heterogeneity, with one sample collected from the intramural area and one from the invasive front area. Previously, Aizawa reported the HER2-overexpression status using the same sets [48]. The kappa statistic was determined to quantify the accordance of the HER2 IHC determination (negative, score of 0 and $1+$ or positive, score of $2+$ and $3+)$ between Aizawa's and our evaluation. The kappa statistic for the HER2 determination was 0.923, indicating an excellent accordance. Although the IHC staining status is heterogeneous in gastric cancer, the TMA method can provide meaningful information when used in a large cohort.

In conclusion, our results showed that $63.1 \%$ of GAC patients expressed at least one RTK when examined using an IHC assay and could be candidates for targeted therapies blocking these RTK pathways. On the other hand, $22.7 \%$ of the GACs in these patients, one-third of at least one RTK overexpressing cases, exhibited the simultaneous 
expression of multiple RTKs. This phenomenon may influence the selection of targeted therapies to prevent the development of drug resistance or primary treatment failure. Our results may be useful for selecting the most suitable patients for each targeted therapy.

Acknowledgments This work was partially supported by grants from the Third-Term Comprehensive 10-year Strategy for Cancer Control from the Ministry of Health, Labor and Welfare. We are grateful to Ms Sachiko Fukuda and Ms Yuka Nakamura for their technical assistance.

\section{References}

1. Ministry of Health LaW. Vital Statistics Japan 2010.

2. Matsuda T, Marugame T, Kamo K, Katanoda K, Ajiki W, Sobue T. Cancer incidence and incidence rates in Japan in 2006: based on data from 15 population-based cancer registries in the monitoring of cancer incidence in Japan (MCIJ) project. Jpn J Clin Oncol. 2012;42:139-47.

3. Koizumi W, Narahara H, Hara T, Takagane A, Akiya T, Takagi $\mathrm{M}$, et al. S-1 plus cisplatin versus S-1 alone for first-line treatment of advanced gastric cancer (SPIRITS trial): a phase III trial. Lancet Oncol. 2008;9:215-21.

4. Boku N, Yamamoto S, Fukuda H, Shirao K, Doi T, Sawaki A, et al. Fluorouracil versus combination of irinotecan plus cisplatin versus S-1 in metastatic gastric cancer: a randomised phase 3 study. Lancet Oncol. 2009;10:1063-9.

5. Bang YJ, Van Cutsem E, Feyereislova A, Chung HC, Shen L, Sawaki A, et al. Trastuzumab in combination with chemotherapy versus chemotherapy alone for treatment of HER2-positive advanced gastric or gastro-oesophageal junction cancer (ToGA): a phase 3, open-label, randomised controlled trial. Lancet. 2010;376:687-97.

6. Waddell T, Chau I, Cunningham D, Gonzalez D, Okines AF, Okines $\mathrm{C}$, et al. Epirubicin, oxaliplatin, and capecitabine with or without panitumumab for patients with previously untreated advanced oesophagogastric cancer (REAL3): a randomised, open-label phase 3 trial. Lancet Oncol. 2013;14:481-9.

7. Lordick F, Kang YK, Chung HC, Salman P, Oh SC, Bodoky G, et al. Capecitabine and cisplatin with or without cetuximab for patients with previously untreated advanced gastric cancer (EXPAND): a randomised, open-label phase 3 trial. Lancet Oncol. 2013;14:490-9.

8. Kim YH, Sasaki Y, Lee KH, Rha SY, Park S, Boku N, et al. Randomized phase II study of nimotuzumab, an anti-EGFR antibody, plus irinotecan in patients with 5-fluorouracil-based regimen-refractory advanced or recurrent gastric cancer in Korea and Japan: preliminary results. J Clin Oncol. 2011;29:4 abstr 87.

9. Oliner KS, Tang R, Anderson A, Lan Y, Iveson T, Donehower $\mathrm{RC}$ et al. Evaluation of MET pathway biomarkers in a phase II study of rilotumumab (R, AMG 102) or placebo (P) in combination with epirubicin, cisplatin, and capecitabine (ECX) in patients (pts) with locally advanced or metastatic gastric (G) or esophagogastric junction (EGJ) cancer. J Clin Oncol. 2012; 30(suppl; abstr 4005).

10. Cunningham D, Al-Batran S-E, Davidenko I, Ilson DH, Murad AM, Tebbutt NC et al. RILOMET-1: An international phase III multicenter, randomized, double-blind, placebo-controlled trial of rilotumumab plus epirubicin, cisplatin, and capecitabine (ECX) as first-line therapy in patients with advanced MET-positive gastric or gastroesophageal junction (G/GEJ) adenocarcinoma. J Clin Oncol. 2013;31(suppl; abstr TPS4153).

11. Brooks AN, Kilgour E, Smith PD. Molecular pathways: fibroblast growth factor signaling: a new therapeutic opportunity in cancer. Clin Cancer Res. 2012;18:1855-62.

12. Association JGC. Japanese classification of gastric carcinoma: 3rd English edition. Gastric Cancer. 2011;14:101-12.

13. Hofmann M, Stoss O, Shi D, Buttner R, van de Vijver M, Kim W, et al. Assessment of a HER2 scoring system for gastric cancer: results from a validation study. Histopathology. 2008;52: 797-805.

14. Cappuzzo F, Hirsch FR, Rossi E, Bartolini S, Ceresoli GL, Bemis $\mathrm{L}$, et al. Epidermal growth factor receptor gene and protein and gefitinib sensitivity in non-small-cell lung cancer. J Natl Cancer Inst. 2005;97:643-55.

15. Drescher D, Moehler M, Gockel I, Frerichs K, Muller A, Dunschede $\mathrm{F}$, et al. Coexpression of receptor-tyrosine-kinases in gastric adenocarcinoma-a rationale for a molecular targeting strategy. World J Gastroenterol. 2007;13:3605-9.

16. Guo T, Fan L, Ng WH, Zhu Y, Ho M, Wan WK, et al. Multidimensional identification of tissue biomarkers of gastric cancer. J Proteome Res. 2012;11:3405-13.

17. Nakajima M, Sawada H, Yamada Y, Watanabe A, Tatsumi M, Yamashita J, et al. The prognostic significance of amplification and overexpression of c-met and c-erb B-2 in human gastric carcinomas. Cancer. 1999;85:1894-902.

18. Matsubara J, Yamada Y, Hirashima Y, Takahari D, Okita NT, Kato K, et al. Impact of insulin-like growth factor type 1 receptor, epidermal growth factor receptor, and HER2 expressions on outcomes of patients with gastric cancer. Clin Cancer Res. 2008; 14:3022-9.

19. Liang Z, Zeng X, Gao J, Wu S, Wang P, Shi X, et al. Analysis of EGFR, HER2, and TOP2A gene status and chromosomal polysomy in gastric adenocarcinoma from Chinese patients. BMC Cancer. 2008;8:363.

20. Lennerz JK, Kwak EL, Ackerman A, Michael M, Fox SB, Bergethon $\mathrm{K}$, et al. MET amplification identifies a small and aggressive subgroup of esophagogastric adenocarcinoma with evidence of responsiveness to crizotinib. $\mathrm{J}$ Clin Oncol. 2011;29:4803-10.

21. Deng N, Goh LK, Wang H, Das K, Tao J, Tan IB, et al. A comprehensive survey of genomic alterations in gastric cancer reveals systematic patterns of molecular exclusivity and cooccurrence among distinct therapeutic targets. Gut. 2012;61: 673-84.

22. Jung EJ, Min SY, Kim MA, Kim WH. Fibroblast growth factor receptor 2 gene amplification status and its clinicopathologic significance in gastric carcinoma. Hum Pathol. 2012;43:1559-66.

23. Xie L, Su X, Zhang L, Yin X, Tang L, Zhang X, et al. FGFR2 gene amplification in gastric cancer predicts sensitivity to the selective FGFR inhibitor AZD4547. Clin Cancer Res. 2013; 19:2572-83.

24. Kunz PL, Mojtahed A, Fisher GA, Ford JM, Chang DT, Balise $\mathrm{RR}$, et al. HER2 expression in gastric and gastroesophageal junction adenocarcinoma in a US population: clinicopathologic analysis with proposed approach to HER2 assessment. Appl Immunohistochem Mol Morphol. 2012;20:13-24.

25. Lee HE, Kim MA, Lee HS, Jung EJ, Yang HK, Lee BL, et al. MET in gastric carcinomas: comparison between protein expression and gene copy number and impact on clinical outcome. Br J Cancer. 2012;107:325-33.

26. Kataoka Y, Okabe H, Yoshizawa A, Minamiguchi S, Yoshimura $\mathrm{K}$, Haga $\mathrm{H}$, et al. HER2 expression and its clinicopathological features in resectable gastric cancer. Gastric Cancer. 2013;16:84-93. 
27. Bang YJ. A randomized, open-label, phase III study of lapatinib in combination with weekly paclitaxel versus weekly paclitaxel alone in the second-line treatment of HER2 amplified advanced gastric cancer (AGC) in Asian population: Tytan study. J Clin Oncol. 2012;30(suppl 34; abstr 11).

28. Hecht JR, Bang Y-J, Qin S, Chung H-C, Xu J-M, Park JO et al. Lapatinib in combination with capecitabine plus oxaliplatin (CapeOx) in HER2-positive advanced or metastatic gastric, esophageal, or gastroesophageal adenocarcinoma (AC): The TRIO-013/LOGiC Trial. J. Clin. Oncol. 2013;31(suppl; abstr LBA4001).

29. Corso S, Ghiso E, Cepero V, Sierra JR, Migliore C, Bertotti A, et al. Activation of HER family members in gastric carcinoma cells mediates resistance to MET inhibition. Mol Cancer. 2010;9:121.

30. Lu Y, Zi X, Zhao Y, Mascarenhas D, Pollak M. Insulin-like growth factor-I receptor signaling and resistance to trastuzumab (Herceptin). J Natl Cancer Inst. 2001;93:1852-7.

31. Gallardo A, Lerma E, Escuin D, Tibau A, Munoz J, Ojeda B, et al. Increased signalling of EGFR and IGF1R, and deregulation of PTEN/PI3 K/Akt pathway are related with trastuzumab resistance in HER2 breast carcinomas. Br J Cancer. 2012;106: 1367-73.

32. Shattuck DL, Miller JK, Carraway KL 3rd, Sweeney C. Met receptor contributes to trastuzumab resistance of Her2-overexpressing breast cancer cells. Cancer Res. 2008;68:1471-7.

33. Engelman JA, Zejnullahu K, Mitsudomi T, Song Y, Hyland C, Park JO, et al. MET amplification leads to gefitinib resistance in lung cancer by activating ERBB3 signaling. Science. 2007;316: 1039-43.

34. Bean J, Brennan C, Shih JY, Riely G, Viale A, Wang L, et al. MET amplification occurs with or without T790 M mutations in EGFR mutant lung tumors with acquired resistance to gefitinib or erlotinib. Proc Natl Acad Sci USA. 2007;104:20932-7.

35. Chen CT, Kim H, Liska D, Gao S, Christensen JG, Weiser MR. MET activation mediates resistance to lapatinib inhibition of HER2-amplified gastric cancer cells. Mol Cancer Ther. 2012; 11:660-9.

36. Kim MA, Lee HS, Lee HE, Jeon YK, Yang HK, Kim WH. EGFR in gastric carcinomas: prognostic significance of protein overexpression and high gene copy number. Histopathology. 2008; 52:738-46.

37. Kim KC, Koh YW, Chang HM, Kim TH, Yook JH, Kim BS, et al. Evaluation of HER2 protein expression in gastric carcinomas: comparative analysis of 1,414 cases of whole-tissue sections and 595 cases of tissue microarrays. Ann Surg Oncol. 2011; 18:2833-40.
38. Sawaki A, Ohashi Y, Omuro Y, Satoh T, Hamamoto Y, Boku N, et al. Efficacy of trastuzumab in Japanese patients with HER2positive advanced gastric or gastroesophageal junction cancer: a subgroup analysis of the Trastuzumab for Gastric Cancer (ToGA) study. Gastric Cancer. 2012;15:313-22.

39. Terashima M, Kitada K, Ochiai A, Ichikawa W, Kurahashi I, Sakuramoto S, et al. Impact of expression of human epidermal growth factor receptors EGFR and ERBB2 on survival in stage II/ III gastric cancer. Clin Cancer Res. 2012;18:5992-6000.

40. He C, Bian XY, Ni XZ, Shen DP, Shen YY, Liu H, et al. Correlation of human epidermal growth factor receptor 2 expression with clinicopathological characteristics and prognosis in gastric cancer. World J Gastroenterol. 2013;19:2171-8.

41. Shan L, Ying J, Lu N. HER2 expression and relevant clinicopathological features in gastric and gastroesophageal junction adenocarcinoma in a Chinese population. Diagn Pathol. 2013; 8:76.

42. Lee J, Seo JW, Jun HJ, Ki CS, Park SH, Park YS, et al. Impact of MET amplification on gastric cancer: possible roles as a novel prognostic marker and a potential therapeutic target. Oncol Rep. 2011;25:1517-24.

43. Betts G, Valentine H, Pritchard S, Swindell R, Williams V, Morgan S, et al. FGFR2, HER2 and cMet in gastric adenocarcinoma: detection, prognostic significance and assessment of downstream pathway activation. Virchows Arch. 2014;464: 145-56.

44. Hattori $\mathrm{Y}$, Itoh $\mathrm{H}$, Uchino $\mathrm{S}$, Hosokawa $\mathrm{K}$, Ochiai A, Ino $\mathrm{Y}$, et al. Immunohistochemical detection of K-sam protein in stomach cancer. Clin Cancer Res. 1996;2:1373-81.

45. Toyokawa T, Yashiro M, Hirakawa K. Co-expression of keratinocyte growth factor and $\mathrm{K}$-sam is an independent prognostic factor in gastric carcinoma. Oncol Rep. 2009;21:875-80.

46. Martin AJ, Grant A, Ashfield AM, Palmer CN, Baker L, Quinlan PR, et al. FGFR2 protein expression in breast cancer: nuclear localisation and correlation with patient genotype. BMC Res notes. 2011;4:72.

47. Behrens C, Lin HY, Lee JJ, Raso MG, Hong WK, Wistuba II, et al. Immunohistochemical expression of basic fibroblast growth factor and fibroblast growth factor receptors 1 and 2 in the pathogenesis of lung cancer. Clin Cancer Res. 2008;14:6014-22.

48. Aizawa M, Nagatsuma AK, Kitada K, Kuwata T, Fujii S, Kinoshita T, et al. Evaluation of HER2-based biology in 1,006 cases of gastric cancer in a Japanese population. Gastric Cancer. $2014 ; 17: 34-42$. 